\title{
An Extension of Kotani's Theorem to Random Generalized Sturm-Liouville Operators
}

\author{
Nariyuki Minami \\ Department of Applied Physics, Tokyo Institut of Technology, O-okayama, Meguro, Tokyo 152, Japan
}

\begin{abstract}
We consider the random operator: $-d / m_{\omega}(d x) d^{+} / d x+q_{\omega}(x)$, where $m_{\omega}(d x)$ and $q_{\omega}(x)$ are a stationary ergodic random measure and a random function respectively. To this general case, we extend Kotani's theorem which asserts that the absolutely continuous spectrum is completely determined by the Ljapounov indices. Our framework includes the case of stochastic Jacobi matrices treated by Simon.
\end{abstract}

\section{Introduction}

In [9] Kotani treated the one-dimensional Schrödinger operator with a stationary, ergodic, bounded potential:

$$
-\frac{d^{2}}{d x^{2}}+q_{\omega}(x)
$$

and proved that with probability one its absolutely continuous spectrum coincides, up to a set of Lebesgue measure zero, with the totality of real numbers for which Ljapounov indices vanish. Simon [13] proved that this theorem holds also for stochastic Jacobi matrices of the following type:

$$
H_{\omega} u(n)=-u(n+1)-u(n-1)+V_{\omega}(n) u(n) .
$$

Our aim here is to extend Kotani's theory, which includes the abovementioned theorem, to the random generalized Sturm-Liouville operator:

$$
L_{\omega} u(x)=-\frac{d}{m_{\omega}(d x)} \frac{d^{+} u}{d x}+q_{\omega}(x) u(x)
$$

where $m_{\omega}(d x)$ is a stationary ergodic random Radon measure on $\mathbb{R}^{1} ; d^{+} u / d x=u^{+}(x)$ is the right-derivative, $d / m_{\omega}(d x)$ is the Radon-Nikodym derivative, and $q_{\omega}(x)$ is a bounded random function defined for $x \in \operatorname{Supp}\left(m_{\omega}\right)$. Here $\operatorname{Supp}\left(m_{\omega}\right)$ is the totality of $x$ such that $m_{\omega}((x-\varepsilon, x+\varepsilon))>0$ for any $\varepsilon>0$. It will be shown that most of the results in [9], including the methods of proofs, hold also for this random operator.

This class of operators contains Schrödinger operators with random potentials 
and the Hamiltonian of a vibrating string (when $m_{\omega}$ is continuous) or a harmonic chain (when $m_{\omega}$ consists of discrete point masses) with random mass distributions. In particular, if we take as $m_{\omega}$ the measure which consists of unit point masses distributed with equal spacings, $L_{\omega}$ becomes the stochastic Jacobi matrix treated by Simon [13]. Thus we can treat both continuum- and discrete models simultaneously.

We give below the outline of this paper. We formulate the problem and state the results in Sect. 2. In Sect. 3, we summarize some facts about the eigenfunction expansion in order to prepare for what follows. In Sect. 4, we introduce, just as in Johnson and Moser [6] and Kotani [9], the function $w$ which is defined as the expectation value of the reciprocal of the Green function of $L_{\omega}$. Using this function, we prove our main theorem in Sect. 5. In Sect. 6, we show how the case of stochastic Jacobi matrices is included in the framework of Sturm-Liouville operators. In the last section, Sect. 7, we state some simple facts concerning inverse spectral problems.

\section{Formulation of the Problem and Statement of Results}

Let $(\Omega, \mathscr{F}, P)$ be a complete probability space and let $\left\{T_{x}: x \in \mathbb{R}^{1}\right\}$ be a one parameter group of measure preserving transformations on this probability space which is ergodic. Let $X$ be defined by

$$
\begin{gathered}
X=\left\{(m, q): m \text { is a non-negative Radon measure on } \mathbb{R}^{1}\right. \text { and } \\
q \text { is a measurable function from } \operatorname{Supp}(m) \text { into } \\
[0,1]\} .
\end{gathered}
$$

Two elements $(m, q)$ and $\left(m^{\prime}, q^{\prime}\right)$ will be identified if $m=m^{\prime}$ and $q(x)=q^{\prime}(x), m$-a.e. on $\operatorname{Supp}(m)$. We say that the sequence $\left\{\left(m_{n}, q_{n}\right): n \geqq 1\right\}$ in $X$ converges to $(m, q) \in X$ if two sequences of Radon measures $\left\{m_{n}(d x)\right\}$ and $\left\{q_{n}(x) m_{n}(d x)\right\}$ converge vaguely to $m(d x)$ and $q(x) m(d x)$ respectively. It is easy to check that $X$ becomes a complete separable metrizable space. Let $\mathscr{B}$ be the topological $\sigma$-field on $X$.

Now let $\left(m_{\omega}, q_{\omega}\right)$ be a random variable on $(\Omega, \mathscr{F}, P)$ with values in $X$. We assume that it is stationary in the following sense:

$$
\left(m_{T_{x} \omega}(\cdot), q_{T_{x} \omega}(\cdot)\right)=\left(m_{\omega}(\cdot+x), q_{\omega}(\cdot+x)\right), x \in \mathbb{R}^{1},
$$

and that

$$
\text { (A) } 0<E\left[m_{\omega}([0,1])\right]<\infty .
$$

From these conditions and the individual ergodic theorem, we see that with probability one

$$
m_{\omega}((0, x]) \sim \mu x \text { and } m_{\omega}([-x, 0]) \sim \mu x, \text { as } x \rightarrow \infty,
$$

where $\mu=E\left[m_{\omega}([0,1])\right]$.

Now for each $\omega \in \Omega$, let $L_{\omega}$ be the generalized Sturm-Liouville operator defined by (1.1). More precisely, we consider the operator $L_{\omega}$ to act on a function $f \in L^{2}$ $\left(\mathbb{R}^{1} ; m_{\omega}\right)$ which has a representation

$$
f(x)=a+b x+\int_{0}^{x} d \xi \int_{0}^{\xi} g(z) m_{\omega}(d z),
$$


with some $g \in L^{2}\left(\mathbb{R}^{1} ; m_{\omega}\right)$ and some constants $a, b \in \mathbb{C}$, where

$$
\int_{0}^{\xi}=\int_{(0, \xi]} \text { if } \xi>0: \quad \int_{0}^{\xi}=-\int_{(\xi, 0]} \text { if } \xi \leqq 0 .
$$

We denote by $D\left(L_{\omega}\right)$ the totality of such functions and for $f \in D\left(L_{\omega}\right)$ we define $L_{\omega} f=$ $-g+q_{\omega} f$. Note that a function from $D\left(L_{\omega}\right)$ has a constant slope in each interval of $m_{\omega}$-measure zero. From (2.1) and the assumption $q_{\omega} \geqq 0$, we see that with probability one $L_{\omega}$ belongs to Weyl's limit point type at $\pm \infty$, and consequently $\left(L_{\omega}, D\left(L_{\omega}\right)\right)$ is self-adjoint in $L^{2}\left(\mathbb{R}^{1} ; m_{\omega}\right)$ (See e.g. [5].)

Let $\Sigma^{\mathrm{ac}}$ be the essential support of the resolution of the identity of $L_{\omega}$ with respect to the Lebesgue measure (essential a.c. spectrum). It can be shown that there exists a non-random set $\Sigma^{\mathrm{ac}}$ such that for $P$-a.a. $\omega, \Sigma_{\omega}^{\mathrm{ac}}=\Sigma^{\mathrm{ac}}$ up to a set of Lebesgue measure zero (see $[4,1]$ ). Let $\Sigma$ be the spectrum of $L_{\omega}$, which is also independent of $\omega$ as is easily seen from the argument of [12].

Next let us introduce the notion of the Ljapounov indices. Let $\phi_{1}=\phi_{1}(x, \lambda ; \omega)$ and $\phi_{2}=\phi_{2}(x, \lambda ; \omega)$ be unique solutions of the integral equation

$$
u(x)=a+b x+\int_{0}^{x} d \xi \int_{0}^{\xi}\left(q_{\omega}(z)-\lambda\right) u(z) m_{\omega}(d z)
$$

corresponding to the initial values $(a, b)=(1,0)$ and $(0,1)$ respectively. The integral Eq. (2.3) is written in short

$$
L_{\omega} u=\lambda u, \quad u(0)=a, \quad u^{+}(0)=b .
$$

For each $\lambda \in \mathbb{C}$, the Ljapounov index $\gamma(\lambda)$ is by definition the following nonnegative limit:

$$
\gamma(\lambda)=\lim _{x \rightarrow \pm \infty} \frac{1}{|x|} \log \left\|M_{\lambda}(x ; \omega)\right\|
$$

where

$$
M_{\lambda}(x ; \omega)=\left[\begin{array}{cc}
\phi_{1}(x, \lambda ; \omega) & \phi_{2}(x, \lambda ; \omega) \\
\phi_{1}^{+}(x, \lambda ; \omega) & \phi_{2}^{+}(x, \lambda ; \omega)
\end{array}\right] .
$$

The almost sure existence and the non-randomness of this limit are guaranteed by Kingman's subadditive ergodic theorem and the assumption of ergodicity ([8]).

Now our results are the following.

Theorem 1. $\Sigma^{\mathrm{ac}}=\left\{\xi \in \mathbb{R}^{1}: \gamma(\xi)=0\right\}$ up to a set of Lebesgue measure zero.

Theorem 2. If $\gamma(\xi)=0$ a.e. on an interval $I \subset \mathbb{R}^{1}$, then with probability one the spectrum of $L_{\omega}$ is purely absolutely continuous on $I$.

Theorem 3. Assume that $q_{\omega}=0$. If the Lebesgue measure of the set $\left\{\xi \in \mathbb{R}^{1}: \gamma(\xi)=0\right\}$ is positive, then the random Radon measure $\left\{m_{\omega}\right\}$ is deterministic in the following sense: up to sets of P-measure zero, $\mathscr{B}_{-\infty}=\mathscr{B}=\mathscr{B}_{\infty}$, where $\mathscr{B}=\sigma\left[\left\langle m_{\omega}, \phi\right\rangle: \phi \in C_{0}\left(\mathbb{R}^{1}\right)\right]$, $\mathscr{B}_{-\infty}=\bigcap_{t} \sigma\left[\left\langle m_{\omega}, \phi\right\rangle: \phi \in C_{0}((-\infty, t])\right]$ and $\mathscr{B}_{\infty}=\bigcap_{t} \sigma\left[\left\langle m_{\omega}, \phi\right\rangle: \phi \in C_{0}((t, \infty))\right]$.

Remark. Theorems 1 and 2 above are extensions of Kotani's Theorems 4.1 and 4.2 
[9] respectively, and include Simon's Theorems 1 and 2 [13] as corollaries.

Theorem 3 is an analogue of Kotani's Theorem 4.3 and Simon's Theorem 3, in which they treat the case where $m_{\omega}$ is fixed but $q_{\omega}$ is random. In the present case, $q_{\omega}$ is constant and $m_{\omega}$ is random. The author could not see what can be said about the determinicity when both $m_{\omega}$ and $q_{\omega}$ are random.

\section{Eigenfunction Expansion of the Green Function}

As we noted in Sect. 2, $L_{\omega}$ belongs to Weyl's limit point type at $\pm \infty$ for $P$-almost all $\omega$, and for such $\omega$, it is well known that the limits

$$
h_{ \pm}(\lambda ; \omega)=\mp \lim _{x \rightarrow \pm \infty} \phi_{1}(x, \lambda ; \omega) / \phi_{2}(x, \lambda ; \omega),
$$

exist for $\lambda \in \mathbb{C} \backslash[0, \infty)$ and define holomorphic functions there. Moreover $\operatorname{Im} \lambda$ $\operatorname{Im} h_{ \pm}(\lambda ; \omega)>0$ for $\operatorname{Im} \lambda \neq 0$ and $h_{ \pm}(\lambda ; \omega)<0$ for $\lambda<0$. For details about such a class of functions, see Kac and Krein [7].

For each $\lambda \in \mathbb{C} \backslash[0, \infty)$,

$$
f_{ \pm}(x)=f_{ \pm}(x, \lambda ; \omega)=\phi_{1}(x) \pm h_{ \pm}(\lambda) \phi_{2}(x),
$$

are solutions of $L_{\omega} u=\lambda u$, which belong to $L^{2}\left(R_{ \pm} ; m_{\omega}\right)$ respectively, where $\mathbb{R}_{+}=$ $(0, \infty)$ and $\mathbb{R}_{-}=(-\infty, 0]$. For $\lambda<0, f_{+}(x)\left[f_{-}(x)\right]$ is positive and decreasing [increasing] in $x$. For $\operatorname{Im} \lambda \neq 0$, we have

$$
\int_{\mathbb{R}_{ \pm}}\left|f_{ \pm}(x, \lambda ; \omega)\right|^{2} m_{\omega}(d x)=\operatorname{Im} h_{ \pm}(\lambda ; \omega) / \operatorname{Im} \lambda
$$

Now for each $\lambda \in \mathbb{C} \backslash[0, \infty)$,

$$
g_{\lambda}(x, y ; \omega)=g_{\lambda}(y, x ; \omega)=-\left(h_{+}+h_{-}\right)^{-1} f_{-}(x) f_{+}(y), \quad x \leqq y,
$$

is an integral kernel of the resolvent $\left(L_{\omega}-\lambda\right)^{-1}$ and will be called the Green function. As an integral kernel of an operator, the value of $g_{\lambda}(x, y ; \omega)$ is meaningless if either $x$ or $y$ does not belong to $\operatorname{Supp}\left(m_{\omega}\right)$. But we take (3.3) as the definition of the Green function, and consider $g_{\lambda}$ to be defined not only for $m_{\omega}$-a.a. $x$ and $y$ but also for all $x$, $y \in \mathbb{R}^{1}$. As is well known [11], there exists a symmetric and non-negative definite measure matrix $\left\{\sigma_{i j}(d \xi ; \omega)\right\}_{i, j=1}^{2}$ such that

$$
g_{\lambda}(x, y ; \omega)=\int_{0-}^{\infty} \frac{1}{\xi-\lambda} \sum_{i, j=1}^{2} \phi_{i}(x, \lambda ; \omega) \phi_{j}(y, \lambda ; \omega) \sigma_{i j}(d \xi ; \omega)
$$

for all $x, y \in \operatorname{Supp}\left(m_{\omega}\right)$. For general $x, y \in \mathbb{R}^{1}$, this equality fails. But if we denote by $\tilde{g}_{\lambda}(x, y ; \omega)$ the right-hand side of (3.4) (which is well defined for all $\left.x, y \in \mathbb{R}^{1}\right)$, then we can prove

$$
g_{\lambda}(x, y ; \omega)=\tilde{g}_{\lambda}(x, y ; \omega)+\Phi_{\omega}(x, y), \text { all } x, y \in \mathbb{R}^{1},
$$

where $\Phi_{\omega}(x, y)$ is non-negative and symmetric in $x, y$, and takes non-zero values if and only if $x$ and $y$ belong to the same interval of $m_{\omega}$-measure zero (compare Dym and McKean [5], Sect. 5.5 Exercise 2). Noting this property of $\Phi_{\omega}$ and the fact that any solution of $L_{\omega} u=\lambda u$ has a constant slope on each interval of $m_{\omega}$-measure zero, 
we see that the resolvent equation

$$
g_{\lambda}(x, y ; \omega)-g_{\mu}(x, y ; \omega)-(\lambda-\mu) \int_{-\infty}^{\infty} g_{\lambda}(x, z ; \omega) g_{\mu}(z, y ; \omega) m_{\omega}(d z)=0,
$$

holds for all $x, y \in \mathbb{R}^{1}$.

Under these remarks, it is easy to check that almost all formulas in Sect. 1 of [9] hold also in our case. In particular we have the following:

$z_{ \pm}(x)=h_{ \pm}\left(\lambda ; T_{x} \omega\right)$ satisfy the Riccati equations:

$$
\begin{gathered}
d z_{ \pm}(x)= \pm\left[\left(q_{\omega}(x)-\lambda\right) m_{\omega}(d x)-z_{ \pm}(x)^{2} d x\right], \\
g_{\lambda}(x, x ; \omega)=g_{\lambda}\left(0,0 ; T_{x} \omega\right), \\
h_{+}\left(\lambda ; T_{x} \omega\right)-h_{-}\left(\lambda ; T_{x} \omega\right)=\frac{d^{+}}{d x} \log g_{\lambda}(x, x ; \omega), \\
g_{\lambda}(x, x ; \omega) m_{\omega}(d x)+\left(\frac{d}{d \lambda} \frac{1}{2 g_{\lambda}(x, x ; \omega)}\right) d x=-d\left(H\left(T_{x} \omega\right)\right),
\end{gathered}
$$

where

$$
H_{\lambda}(\omega)=\frac{1}{2} g_{\lambda}(0,0 ; \omega)\left\{\int_{R_{+}} f_{+}(x, \lambda ; \omega)^{2} m_{\omega}(d x)-\int_{R_{-}} f_{-}(x, \lambda ; \omega)^{2} m_{\omega}(d x)\right\} .
$$

In order to deduce (3.10), we have used the resolvent equation (3.6) with $x=y$, where $x$ does not necessarily belong to $\operatorname{Supp}\left(m_{\omega}\right)$.

\section{The w-Function and its Properties}

In this section, we will show that the function

$$
w(\lambda)=\frac{1}{2} E\left[h_{+}(\lambda ; \omega)+h_{-}(\lambda ; \omega)\right]=-\frac{1}{2} E\left[\frac{1}{g_{\lambda}(0,0 ; \omega)}\right]
$$

is well defined for any $\lambda \in \mathbb{C} \backslash[0, \infty)$, and derive some of its properties. Such a function was first introduced by Johnson and Moser [6] in the case of almost periodic potentials and was extensively used by Kotani [9] in the case of general stationary ergodic potentials.

Lemma 4.1. For any compact $K \subset \mathbb{C} \backslash[0, \infty),\left\{h_{ \pm}(\lambda ; \omega): \lambda \in K\right\}$ is uniformly integrable with respect to $P$, and consequently $w$ is holomorphic in $\mathbb{C} \backslash[0, \infty)$, satisfies $\operatorname{Im} w(\lambda) \operatorname{Im} \lambda>0$ for $\operatorname{Im} \lambda \neq 0$ and $w(\lambda)<0$ for $\lambda<0$.

Proof. As is done in Kotani [9] (Lemma 1.2), it suffices to estimate $h_{ \pm}(\lambda ; \omega)$ for $\lambda=$ - 1. Moreover the assumption of uniform boundedness of $q_{\omega}(x)$ and a simple comparison argument reduce the problem to the case of $q_{\omega}(x) \equiv 1$, or what is the same, $q_{\omega}(x) \equiv 0$. Now if $q_{\omega} \equiv 0$ and $\lambda<0$, then we see from the inequality of I.S. Kac,

$$
\left|h_{+}(\lambda ; \omega)\right| \leqq|\lambda| m_{\omega}([0, x])+1 / x, x>0 \text {. }
$$

(See [10], Corollary of Theorem 2.2 with $h(\lambda)$ there being replaced by $-h_{ \pm}(-\lambda)^{-1}$.) This inequality combined with the assumption (A) immediately implies the first half of the Lemma. 
From the Cauchy integral representation of $(d / d \lambda) h_{+}(\lambda)$, it is clear that $\left\{(d / d \lambda) h_{ \pm}(\lambda: \omega): \lambda \in K\right\}$ is also uniformly integrable for each compact $K \subset \mathbb{C} \backslash[0, \infty)$. This proves the second half of the lemma.

Lemma 4.2. $w(\lambda)=E\left[h_{ \pm}(\lambda: \omega)\right]$, for any $\lambda \in \mathbb{C} \backslash[0, \infty)$.

Proof. Integrating (3.9) with respect to $x$ on the interval $I=(0,1)$ and noting (3.8), we have

$$
\int_{0}^{1} h_{+}\left(\lambda ; T_{x} \omega\right) d x-\int_{0}^{1} h_{-}\left(\lambda ; T_{x} \omega\right) d x=\log g_{\lambda}\left(0,0 ; T_{1} \omega\right)-\log g_{\lambda}(0,0 ; \omega) .
$$

Now set $X(\omega)=\log g_{\lambda}(0,0 ; \omega)$ and $Y(\omega)=X\left(T_{1} \omega\right)-X(\omega)$. Lemma 4.1 implies that the left-hand side of $(4.3)$ belongs to $L^{1}(\Omega, P)$ and hence $Y(\omega) \in L^{1}$. We will prove $E[Y]=0$.

To this end set $X_{n}=\min \{n, \max (\dot{X},(-n))\}, Y_{n}(\omega)=X_{n}\left(T_{1} \omega\right)-X_{n}(\omega)$ and $A_{n}=\left\{\omega: \max \left(|X(\omega)|,\left|X\left(T_{1} \omega\right)\right|\right)>n\right\}$. Note that $Y=Y_{n}$ on $A_{n}^{c}$ and $\left|Y_{n}\right| \leqq|Y|$. From these together with the stationarity of $P$, it follows that

$$
|E[Y]|=\left|E\left[Y: A_{n}\right]+E\left[Y_{n}: A_{n}^{c}\right]\right|=\left|E\left[Y: A_{n}\right]-E\left[Y_{n}: A_{n}\right]\right| \leqq 2 E\left[|Y|: A_{n}\right] .
$$

Letting $n \rightarrow \infty$, we obtain $|E[Y]|=0$.

Lemma 4.3. $(d w / d \lambda)(\lambda)=E\left[\int_{(0,1]} g_{\lambda}(x, x ; \omega) m_{\omega}(d x)\right]$ for each $\lambda \in \mathbb{C} \backslash[0, \infty)$.

Proof. Integrating both sides of (3.10) with respect to $x$ on the interval $(0,1]$, and using (3.8), we have

$$
\int_{(0,1]} g_{\lambda}(x, x ; \omega) m_{\omega}(d x)+\int_{0}^{1} \frac{d}{d \lambda}\left(\frac{1}{2 g_{\lambda}\left(0,0 ; T_{x} \omega\right)}\right) d x=-H_{\lambda}\left(T_{1} \omega\right)+H_{\lambda}(\omega) .
$$

Note that $H_{\lambda}\left(T_{x} \omega\right)$ is right-continuous. For each $\lambda \in \mathbb{C} \backslash[0, \infty), H_{\lambda}(\omega)$ is integrable with respect to $P$. Indeed if $\operatorname{Im} \lambda \neq 0$, then we have from (3.2), (3.3) and the definition of $H_{\lambda}(\omega)$,

$$
H_{\lambda}(\omega) \leqq \frac{1}{2} \frac{1}{\operatorname{Im}\left(h_{+}+h_{-}\right)(\lambda ; \omega)} \frac{\operatorname{Im}\left(h_{+}+h_{-}\right)(\lambda ; \omega)}{\operatorname{Im} \lambda}=(2 \operatorname{Im} \lambda)^{-1}
$$

If $\lambda \in(-\infty, 0)$, then noting that $f_{ \pm}(x, \lambda ; \omega)$ are positive monotone solutions of $L_{\omega} u=\lambda u$ and $f_{ \pm}(0, \lambda ; \omega)=1$, it is easily shown that

$$
\int_{R_{ \pm}} f_{ \pm}(x, \lambda ; \omega)^{2} d m_{\omega}(x) \leqq h_{ \pm}(\lambda ; \omega) / \lambda,
$$

and hence $H_{\lambda}(\omega) \leqq(2|\lambda|)^{-1}$.

Now the lemma will be proved if we take expectation of (4.4) and use the stationarity of $P$. Note that from Lemma 4.1, it follows that $\left\{g_{\lambda}\left(0,0 ; T_{x} \omega\right)^{-1}: \lambda \in K\right\}$ is uniformly integrable with respect to $P \times 1_{(0,1]}(x) d x$ for every compact $K \subset C \backslash[0, \infty)$ and that the order of integration and differentiation can be exchanged.

From this lemma and the eigenfunction expansion (3.4), we see that $d w / d \lambda$ has 
the following expression:

$$
\frac{d w}{d \lambda}=\int_{0}^{\infty} \frac{1}{\xi-\lambda} d n(\xi)
$$

where we set

$$
n(\xi)=E\left[\int_{(0,1]} m_{\omega}(d x) \sigma_{11}\left([0, \xi] ; T_{x} \omega\right)\right] .
$$

This $n(\xi)$ can be identified with the integrated density of states. That is, if we restrict $L_{\omega}$ to a finite interval $I$ and impose a self-adjoint boundary condition, and if we denote by $N_{I}(\xi ; \omega)$ the number of eigenvalues $\leqq \xi$ of this eigenvalue problem, then it can be shown that for any $\xi \in \mathbb{R}^{1}$,

$$
n(\xi)=\lim _{I \uparrow \mathbb{R}^{\mid}} \frac{1}{|I|} N_{I}(\xi ; \omega),
$$

almost surely. But since this fact will not be used in the following, we will not discuss its details.

Lemma 4.4. It holds that for any $\lambda \in \mathbb{C}_{+}=\{\operatorname{Im} \lambda>0\}$,

$$
-2 \operatorname{Re} w(\lambda)=E\left[\operatorname{Im} \lambda \int_{0}^{1} \frac{m^{c}(d x)}{\operatorname{Im} h_{ \pm}} \pm \sum_{x \in(0,1]} \log \left(\frac{\operatorname{Im} h_{ \pm} \pm \operatorname{Im} \lambda \mathrm{m}[x]}{\operatorname{Im} h_{ \pm}}\right)\right],
$$

where $m^{c}=m_{\omega}^{c}$ is the continuous part of $m_{\omega}, m[x]=m_{\omega}(\{x\})$ and $\operatorname{Im} h_{ \pm}$is an abbreviation of $\operatorname{Im} h_{ \pm}\left(\lambda ; T_{x} \omega\right)$.

Proof. We quote the following simple lemma: for any real valued, right-continuous function $f$ on $(a, b]$ which is of bounded variation, and for any function $F$ which is smooth on the range of $f$,

$$
F(f(b))-F(f(a))=\int_{(a, b]} F^{\prime}(f(x)) d f^{c}(x)+\sum_{x \in(a, b]}\{F(f(x))-F(f(x-))\},
$$

where $d f^{c}$ is the continuous part of the signed Stieltjes measure $d f$. Now set $Y_{ \pm}(x)=$ $\operatorname{Im} h_{ \pm}\left(\lambda ; T_{x} \omega\right), X_{ \pm}(x)=\operatorname{Re} h_{ \pm}\left(\lambda ; T_{x} \omega\right)$ and fix an arbitrary $\delta>0$. If we take the imaginary part of Riccati equation (3.7), divide both sides by $Y_{+}(x)+\delta$ and integrate on $(0,1]$, we obtain

$$
-2 \int_{0}^{1} X_{+}(x) \frac{Y_{+}(x)}{Y_{+}(x)+\delta} d x=\operatorname{Im} \lambda \int_{(0,1]} \frac{m(d x)}{Y_{+}(x)+\delta}+\int_{(0,1]} \frac{d Y_{+}(x)}{Y_{+}(x)+\delta} .
$$

Note that $Y_{ \pm}(x)$ is right-continuous. From (3.7) we also have $Y_{+}(x)-Y_{+}(x-)=$ $-\operatorname{Im} \lambda m[x]$. Using (4.8) with $F(x)=\log (x+\delta)$ in order to evaluate the second term on the right-hand side of (4.9), we arrive at

$$
\begin{aligned}
-2 \int_{0}^{1} X_{+}(x) \frac{Y_{+}(x)}{Y_{+}(x)+\delta} d x= & \log \frac{Y_{+}(1)+\delta}{Y_{+}(0)+\delta}+\sum_{x \in(0,1]} \log \frac{Y_{+}(x)+\operatorname{Im} \lambda m[x]+\delta}{Y_{+}(x)+\delta} \\
& +\operatorname{Im} \lambda \int_{0}^{1} \frac{m^{c}(d x)}{Y_{+}(x)+\delta}
\end{aligned}
$$


Similar argument shows

$$
\begin{aligned}
-2 \int_{0}^{1} X_{-}(x) \frac{Y_{-}(x)}{Y_{-}(x)+\delta} d x= & -\log \frac{Y_{-}(1)+\delta}{Y_{-}(0)+\delta}+\sum_{x \in(0,1]} \log \frac{Y_{-}(x)+\delta}{Y_{-}(x)-\operatorname{Im} \lambda m[x]+\delta} \\
& +\operatorname{Im} \lambda \int_{0}^{1} \frac{m^{c}(d x)}{Y_{-}(x)+\delta} .
\end{aligned}
$$

Note that in this case $Y_{-}(x)-\operatorname{Im} \lambda m[x]=Y_{-}(x-) \geqq 0$. We can take the expectation of each term of (4.10) and (4.11). Each of the first terms on the right-hand side of (4.10) and (4.11) vanish because of the stationarity. Finally letting $\delta \downarrow 0$ and using monotone convergence theorem and Lemmas 4.1 and 4.2, we obtain (4.7).

From these lemmas and remarks, we see that all the arguments in Sects. 2 and 3 in [9] concerning the representation and boundary values of $w(\lambda)$ remain valid also in our case. As for the relation between $w(\lambda)$ and the Ljapounov index $\gamma(\lambda)$, we have even the following:

$$
-\operatorname{Re} w(\xi+i 0)=\gamma(\xi) \text { for all } \xi \in \mathbb{R}^{1} \text { (not only for a.e. } \xi \text { ). }
$$

This follows from the subharmonicity of functions $-\operatorname{Re} w(\lambda)$ and $\gamma(\lambda)$ (see [3]). Equation (4.12) was communicated to the author by Kotani.

Moreover a part of the argument in Lemma 4.1 of [9] remains valid and we have the following: for any compact $K \subset \mathbb{R}^{1}$ on which $\gamma(\xi)=0$, a.e., it holds that

$$
-\lim _{\varepsilon \downarrow 0} \int_{K}(\operatorname{Re} w(\xi+i \varepsilon) / \varepsilon) d \xi=\int_{K} n_{\mathrm{ac}}(\xi) d \xi,
$$

where $n_{\mathrm{ac}}(\xi)$ is the density of the absolutely continuous part of $d n(\xi)$.

Thus we have prepared everything which is necessary to prove Theorem 1. The following inequality will be shown in the proof of Lemma 5.2:

$$
-\frac{\operatorname{Re} w(\lambda)}{\operatorname{Im} \lambda}-\operatorname{Im} \frac{d w}{d \lambda} \geqq 0 \quad \text { for } \lambda \in \mathbb{C}_{+} .
$$

This combined with the argument in Lemma 4.1 of [9] shows

$$
\lim _{\varepsilon \downarrow 0} \int_{K} \operatorname{Im} \frac{d w}{d \lambda}(\xi+i \varepsilon) d \xi=\int_{K} n_{\mathrm{ac}}(\xi) d \xi,
$$

where $K$ and $n_{\mathrm{ac}}$ are as in (4.13). This will be used in the proofs of Theorems 2 and 3 .

\section{Proof of Theorems}

\section{Proof of Theorem 1.}

1) Proof of $A \equiv\left\{\xi \in \mathbb{R}^{1}: \gamma(\xi)=0\right\} \subset \Sigma^{\text {ac }}$ up to Lebesgue measure zero.

For each $\delta>0$, choose $N>0$ so large that we have

$$
P\left(\omega: m_{\omega}((-N, N])>0\right)>1-\delta .
$$

Using the elementary inequality $\log (1+x) \geqq x /(1+x)(x \geqq 0)$, Lemma 4.4 and the 
stationarity of $P$, we get for $\lambda \in \mathbb{C}_{+}$

$$
\frac{1}{2 N} E\left[\int_{(-N, N]}\left\{\frac{1}{\operatorname{Im} h_{-}}+\frac{1}{\operatorname{Im} h_{+}+\operatorname{Im} \lambda m[m]}\right\} m(d x)\right] \leqq \frac{-4 \operatorname{Re} w(\lambda)}{\operatorname{Im} \lambda} .
$$

Setting $\lambda=\xi+i \varepsilon$, integrating (5.1) with respect to $\xi$ on a compact set $K \subset A$ and letting $\varepsilon \downarrow 0$, we have from (4.13) and Fatou's lemma

$$
E\left[\int_{(N, N]} m_{\omega}(d x) \int_{K} d \xi\left\{\frac{1}{\operatorname{Im} h_{+}}+\frac{1}{\operatorname{Im} h_{-}}\right\}\left(\xi+i 0 ; T_{x} \omega\right)\right] \leqq 4 N \int_{K} n_{\mathrm{ac}}(\xi) d \xi<\infty .
$$

Since $K \subset A$ and $\delta>0$ are arbitrary, we arrive at the following assertion: for $P$-a.a $\omega \in \Omega$ and $m_{\omega}$-a.a $x \in \mathbb{R}^{1}$,

$$
\operatorname{Im} h_{ \pm}\left(\xi+i 0 ; T_{x} \omega\right)>0, \text { a.e. } \xi \in A .
$$

The rest of the proof is the same as in [9], and we obtain the inclusion of $A$ in the essential a.c. spectrum of $L_{T_{x} \omega}$ for $m_{\omega}$-a.a. $x$. Since $L_{T_{x} \omega}$ and $L_{\omega}$ are unitarily equivalent, we get the same conclusion for $L_{\omega}$.

2) Proof of $A^{c}=\left\{\xi \in \mathbb{R}^{1}: \gamma(\xi)>0\right\} \subset\left(\Sigma^{\mathrm{ac}}\right)^{c}$ up to Lebesgue measure zero.

This follows from the argument of Pastur [12] and the following Lemma, which should be applied to such $\omega$ 's for which (2.1) holds.

Lemma 5.1 Define the spectral measure by $\rho=\sigma_{11}+\sigma_{22}$. If $m([-x, x])=O(x)$ as $x \rightarrow \infty$, then for $\rho$-a.e. $\xi \in \mathbb{R}^{1}$, there exists a solution of $L u=\xi u$ such that $u(x)$ and $u^{+}(x)$ are polynomially bounded.

Proof. Let $\tau_{i j}(\xi)$ be the density of $\sigma_{i j}(\xi)$ with respect to $\rho$. Then the eigenfunction expansion of $g_{\lambda}$ is rewritten as

$$
g_{\lambda}(x, y)=\Phi(x, y)+\int_{0-\zeta}^{\infty} \frac{1}{\xi-\lambda}\left\{\sum_{i, j=1}^{2} \tau_{i j}(\xi) \phi_{i}(x, \xi) \phi_{j}(y, \xi)\right\} \rho(d \xi)
$$

where $\Phi$ is defined in (3.5). On the other hand, it is easy to show from the monotonicity and positivity of $f_{ \pm}(x, \lambda)$ that for $\lambda<0$

$$
g_{\lambda}(x, x) \leqq g_{\lambda}(0,0)+|x|, x \in \mathbb{R}^{1} .
$$

Therefore from the assumption on $m(d x)$, we have for fixed $\lambda<0$ and $\alpha>2$

$$
\int_{0-}^{\infty} \rho(d \xi) \frac{1}{\xi-\lambda} \int_{-\infty}^{\infty} \frac{1}{1+|x|^{\alpha}}\left\{\sum \tau_{i j}(\xi) \phi_{i}(x, \xi) \phi_{j}(x, \xi)\right\} m(d x)<\infty .
$$

Now set $u(x)=u(x ; y, \omega)=\sum \tau_{i j}(\xi) \phi_{i}(y, \xi) \phi_{j}(x, \xi)$. For each $\xi$ and $y, u(x)$ is a solution of $L u=\xi u$. Since $\left\{\tau_{i j}\right\}$ is non-negative definite, we see from Schwarz's inequality

$$
\int_{-\infty}^{\infty} \frac{\rho(d \xi)}{\xi-\lambda} \int_{-\infty}^{\infty} \frac{d y}{1+|y|^{\alpha}} \int_{-\infty}^{\infty} \frac{m(d x)}{1+|x|^{\alpha}}|u(x ; y, \xi)|<\infty .
$$

In particular, for $\rho$-a.e. $\xi$ we can find a $y$ such that

$$
\int_{-\infty}^{\infty} \frac{1}{1+|x|^{\alpha}}|u(x)| m(d x)<\infty
$$


From (5.4) and the equation $d u^{+}=(q-\xi) u d m$, we have $\left|u^{+}(x)\right|=O\left(|x|^{\alpha}\right)$ for large $|x|$, and hence $|u(x)|=O\left(|x|^{\alpha+1}\right)$ for large $|x|$.

The proofs of Theorem 2 and 3 are based on the following lemma.

Lemma 5.2. Assume that $A=\left\{\xi \in \mathbb{R}^{1}: \gamma(\xi)=0\right\}$ has positive Lebesgue measure. Then for $P$ a.e. $\omega \in \Omega$ and $m_{\omega}$-a.e. $x \in \mathbb{R}^{1}$ it holds

$$
h_{-}\left(\xi+i 0 ; T_{x} \omega\right)=-\overline{h_{+}\left(\xi+i 0 ; T_{x} \omega\right)}, \text { a.e. } \xi \in A .
$$

Proof. Set $\lambda=\xi+i \varepsilon, \quad b=b_{ \pm}(\xi, \varepsilon, x)=\operatorname{Im} h_{ \pm}\left(\xi+i \varepsilon ; T_{x} \omega\right) \quad$ and $\quad a=a(\xi, \varepsilon, x)=$ $\operatorname{Re}\left(h_{+}+h_{-}\right)\left(\xi+i \varepsilon ; T_{x} \omega\right)$. Then we have from Lemmas 4.3 and 4.4,

$$
\begin{aligned}
\int_{K}\{- & \left.\frac{\operatorname{Re} w(\xi+i \varepsilon)}{\varepsilon}-\operatorname{Im} w^{\prime}(\xi+i \varepsilon)\right\} d \xi \\
= & E\left[\int_{0}^{1} m_{\omega}^{c}(d x) \int_{K} d \xi\left(\frac{1}{b_{+}}+\frac{1}{b_{-}}\right) \frac{a^{2}+\left(b_{+}-b_{-}\right)^{2}}{a^{2}+\left(b_{+}+b_{-}\right)^{2}}\right] \\
+ & E\left[\sum_{x \in(0,1)} \int_{K} d \xi m_{\omega}[x] \Gamma\left(b_{+}, b_{-}, a^{2}, \varepsilon m_{\omega}[x]\right)\right],
\end{aligned}
$$

where $K \subset A$ is compact and we have set

$$
\Gamma(s, t, u, \delta)=\frac{1}{4 \delta}\left(\log \frac{s+\delta}{s}+\log \frac{t}{t-\delta}\right)-\frac{s+t}{u+(s+t)^{2}},
$$

for $s>0, t>\delta>0$, and $u \geqq 0$. Note that from Lemma 4.4 (the integrability of the right-hand side of $(4.7)), b_{-}(\xi, \varepsilon, x)-\varepsilon m_{\omega}[x]>0$ almost surely. It holds that $\Gamma \geqq 0$ for such $s, t, u$ and $\delta$. Indeed we can set $u=0$, and in this case the assertion follows from $\Gamma(s, t, 0,0+) \geqq 0$ and $\partial / \partial \delta[4 \delta \Gamma(s, t, 0, \delta)] \geqq 0$. Therefore the integrand of the right-hand side of (5.5) is non-negative, so that we obtain (4.14) and (4.15) as was already remarked. This together with (4.13) implies that the left-hand side of (5.5) tends to zero as $\varepsilon \downarrow 0$. On the other hand from (5.3), $b_{ \pm}(\xi, 0+, x)>0$ a.e. $\xi \in K$ and for such $\xi$,

$$
\lim _{\varepsilon \downarrow 0} \Gamma\left(b_{+}, b_{-}, a^{2}, \varepsilon m_{\omega}[x]\right)=\left(\frac{1}{b_{+}}+\frac{1}{b_{-}}\right) \frac{a^{2}+\left(b_{+}-b_{-}\right)^{2}}{a^{2}+\left(b_{+}+b_{-}\right)^{2}} .
$$

Now let $\varepsilon \downarrow 0$ in (5.5) and use Fatou's lemma. The proof will be complete if we use the stationarity of $P$ and let $K \uparrow A$.

Proof of Theorem 2. Fix $\omega$ and $x$ for which the relation in Lemma 5.2 holds. We see that for a.e. $\xi \in I$,

$$
\operatorname{Re}\left\{-\left(h_{+}+h_{-}\right)^{-1}\left(\xi+i 0 ; T_{x} \omega\right)\right\}=0
$$

and

$$
\operatorname{Re}\left\{h_{+} h_{-} /\left(h_{+}+h_{-}\right)\left(\xi+i 0 ; T_{x} \omega\right)\right\}=0 .
$$

These imply that $-\left(h_{+}+h_{-}\right)^{-1}$ and $h_{+} h_{-} /\left(h_{+}+h_{-}\right)$have analytic continuations to

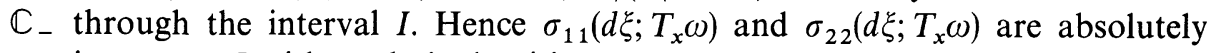
continuous on $I$ with analytic densities. 
Proof of Theorem 3. Let $\Omega^{\prime}$ be the totality of $\omega$ for which the relation in Lemma 5.2 holds. For $t \in \mathbb{R}^{1}$ and $A \in \mathscr{B}$, put $A_{t}^{\prime}=\left\{\left.m_{\omega}\right|_{(-\infty, t]}: \omega \subset A \cap \Omega^{\prime}\right\}$. Then the set $B_{t}=$ $\left\{\omega \in \Omega:\left.m_{\omega}\right|_{(-\infty, t]} \in A_{t}^{\prime}\right\}$ belongs to $\mathscr{B}_{t}=\sigma\left[\left\langle m_{\omega}, \phi\right\rangle: \phi \in C_{0}((-\infty, t])\right]$. Let $\omega \in$ $B_{t} \cap \Omega^{\prime}$. Then there exists $\tilde{\omega} \in A \cap \Omega^{\prime}$ such that the restriction of $m_{\omega}$ to $(-\infty, t]$ coincides with that of $m_{\tilde{\omega}}$. Since we can assume that $\operatorname{Supp}\left(m_{\omega}\right)$ and $\operatorname{Supp}\left(m_{\tilde{\omega}}\right)$ are unbounded from below, $x=\sup \left[(-\infty, t] \cap \operatorname{Supp}\left(m_{\omega}\right) \cap \operatorname{Supp}\left(m_{\tilde{\mathbb{u}}}\right)\right]$ is well defined and it holds that $\left.m_{\omega}\right|_{(-\infty, x]}=\left.m_{\tilde{\omega}}\right|_{(-\infty, x]}$. Hence $h_{-}\left(\lambda ; T_{x} \omega\right) \equiv h_{-}\left(\lambda ; T_{x} \tilde{\omega}\right)$, so that we have from Lemma $5.2 h_{+}\left(\lambda ; T_{x} \omega\right) \equiv h_{+}\left(\lambda ; T_{x} \tilde{\omega}\right)$ on $\mathbb{C}_{+}$, because a function which is holomorphic on $\mathbb{C}_{+}$and which has positive imaginary part is uniquely determined by its boundary values on a subset $\subset \mathbb{R}^{1}$ of positive Lebesgue measure. From the uniqueness of Krein's correspondence (see e.g. [5]), we get $\left.m_{\omega}\right|_{(x, \infty)}=\left.m_{T_{x} \omega}\right|_{(0, \infty)}=$ $\left.m_{T_{x} \tilde{\omega}}\right|_{(0, \infty)}=\left.m_{\tilde{\omega}}\right|_{(x, \infty)}$, i.e. $m_{\omega}=m_{\tilde{\omega}}$, hence $\omega \in A$. Since we trivially have $B_{t} \cap \Omega^{\prime} \supset$ $A \cap \Omega^{\prime}$, and since $P\left(\Omega^{\prime}\right)=1$, we conclude $P\left(A_{\Delta} B_{t}\right)=0$ : hence $\mathscr{B}_{t}=\mathscr{B}$ a.s. and $\mathscr{B}_{-\infty}=$ $\mathscr{B}$ a.s. Similarly $\mathscr{B}_{+\infty}=\mathscr{B}$ a.s.

\section{An Application to Stochastic Jacobi Matrices}

Let $\left(\Omega^{\prime}, \mathscr{F}^{\prime}, P^{\prime}\right)$ be a probability space and $\theta$ be an invertible measure preserving ergodic transformation on it. From random variables $M>0, K>0,0 \leqq Q \leqq 1$ on $\Omega^{\prime}$, we obtain a stationary ergodic sequence of random variables: $\left\{\left(M\left(\theta^{n} \omega^{\prime}\right), K\left(\theta^{n} \omega^{\prime}\right)\right.\right.$, $\left.\left.Q\left(\theta^{n} \omega^{\prime}\right)\right)\right\}$. To each $\omega^{\prime} \in \Omega^{\prime}$, we associate a self-adjoint operator (Jacobi matrix) on $l^{2}\left(\mathbb{Z}^{1}:\left\{M\left(\theta^{n} \omega^{\prime}\right)\right\}\right)$ defined by

$$
\left(L_{\omega}, u\right)_{n}=-\frac{1}{M\left(\theta^{n} \omega^{\prime}\right)}\left\{K\left(\theta^{n} \omega^{\prime}\right)\left(u_{n+1}-u_{n}\right)-K\left(\theta^{n-1} \omega^{\prime}\right)\left(u_{n}-u_{n-1}\right)\right\}+Q\left(\theta^{n} \omega^{\prime}\right) u_{n} .
$$

As before, the spectrum and essential a.c. spectrum are constants for $P^{\prime}$-a.a. $\omega^{\prime}$, and we denote them by $\sigma$ and $\sigma^{\text {a.c. }}$ respectively. We assume

$$
\left(A^{\prime}\right) c \equiv \int K\left(\omega^{\prime}\right)^{-1} P^{\prime}\left(d \omega^{\prime}\right)<\infty, \int K\left(\omega^{\prime}\right) P^{\prime}\left(d \omega^{\prime}\right)<\infty, 0<\int M\left(\omega^{\prime}\right) P^{\prime}\left(d \omega^{\prime}\right)<\infty .
$$

The Ljapounov index $\gamma^{\prime}(\lambda)$ is defined for each $\lambda \in \mathbb{C}$ by the following limit which exists almost surely:

where

$$
\gamma^{\prime}(\lambda)=\lim _{n \rightarrow \pm \infty} \frac{1}{|n|} \log \left\|M_{\lambda}^{\prime}(n ; \omega)\right\|
$$

$$
M_{\lambda}^{\prime}(n ; \omega)=\left[\begin{array}{cc}
u_{1}(n+1) & u_{2}(n+1) \\
u_{1}(n) & u_{2}(n)
\end{array}\right],
$$

and $u_{1}, u_{2}$ are the solutions of $L_{\omega^{\prime}} u=\lambda u$ with $u_{1}(0)=u_{2}(1)=0$ and $u_{1}(1)=u_{2}(0)=1$.

Then we obtain the following results:

Theorem 1'. $\sigma^{\text {a.c. }}=\left\{\xi \in \mathbb{R}^{1}: \gamma^{\prime}(\xi)=0\right\}$ up to a set of Lebesgue measure zero.

Theorem $2^{\prime}$. If $\gamma^{\prime}(\xi)=0$ a.e. on the interval $I \subset \mathbb{R}^{1}$, then with probability one the spectrum of $L_{\omega^{\prime}}$ is purely absolutely continuous on $I$. 
Theorem 3'. Assume that $Q \equiv 0$. If the Lebesgue measure of the set $A^{\prime} \equiv\left\{\xi \in \mathbb{R}^{1}\right.$ : $\left.\gamma^{\prime}(\xi)=0\right\}$ is positive, then the sequence $\left\{\left(M\left(\theta^{n} \omega^{\prime}\right), K\left(\theta^{n} \omega^{\prime}\right)\right): n \in \mathbb{Z}^{1}\right\}$ is deterministic in the following sense: up to sets of $P^{\prime}$-measure zero, $\bigcap_{n} \sigma\left[X_{j}\left(\omega^{\prime}\right): j \leqq n\right]$, $=\sigma\left[X_{j}\left(\omega^{\prime}\right): j \in \mathbb{Z}^{1}\right]=\bigcap_{n} \sigma\left[X_{j}\left(\omega^{\prime}\right): j \geqq n\right]$, where $X_{j}\left(\omega^{\prime}\right)=\left(M\left(\theta^{j} \omega^{\prime}\right), K\left(\theta^{j} \omega^{\prime}\right)\right)$.

In particular, if we take $M \equiv K \equiv 1$ in Theorems $1^{\prime}$ and $2^{\prime}$, we get Theorems 1 and 2 of Simon [13].

We will prove Theorems $1^{\prime}$ and $2^{\prime}$ as corollaries of Theorems 1 and 2 of the previous section. Theorem $3^{\prime}$ will be proved using Lemma 5.2 and the argument parallel to that of Theorem 3.

To this end, let us define a new probability space,

$$
\Omega=\left\{\omega=\left(\omega^{\prime}, s\right): \omega^{\prime} \in \Omega^{\prime}, 0 \leqq s<K\left(\omega^{\prime}\right)^{-1}\right\},
$$

a new $\sigma$-algebra on $\Omega$,

$$
\mathscr{F}=\left\{\mathscr{F}^{\prime} \times \mathscr{B}([0, \infty))\right\} \cap \Omega,
$$

and a new probability measure on $(\Omega, \mathscr{F})$,

$$
P(E)=\frac{1}{c} \iint_{E} d s P^{\prime}\left(d \omega^{\prime}\right), \quad E \in \mathscr{F} .
$$

We define a random Radon measure $m_{\omega}$ on $\mathbb{R}^{1}$ and a random function $q_{\omega}$ by the following formulas:

$$
\begin{aligned}
& m_{\omega}=\sum_{n=-\infty}^{\infty} M\left(\theta^{n} \omega^{\prime}\right) \delta\left(x_{n}(\omega)\right) \\
& q_{\omega}=\sum_{n=-\infty}^{\infty} Q\left(\theta^{n} \omega^{\prime}\right) 1\left(x_{n}(\omega)\right)
\end{aligned}
$$

where

$$
\begin{array}{rlrl}
x_{n}(\omega) & =\sum_{j=0}^{n} K\left(\theta^{j} \omega^{\prime}\right)^{-1}-s \quad & \text { (if } n \geqq 0) \\
& =-\sum_{j=n}^{-1} K\left(\theta^{j} \omega^{\prime}\right)^{-1}-s \quad(\text { if } n<0),
\end{array}
$$

$\delta(x)$ is the unit mass at $x$ and $1(x)$ is the indicator function of $\{x\}$. Then condition (A) follows from condition $\left(\mathrm{A}^{\prime}\right)$ and $L_{\omega^{\prime}}$ defined by (6.1) coincides with

$$
L_{\omega}=-\frac{d}{m_{\omega}(d x)} \frac{d^{+}}{d x}+q_{\omega}(x)
$$

$m_{\omega}$ and $q_{\omega}$ being defined as above.

Now let us define a flow $\left\{T_{x}: x \in \mathbb{R}^{1}\right\}$ on $(\Omega, \mathscr{F}, P)$. For $x \geqq 0$ set

$$
T_{x} \omega=T_{x}\left(\omega^{\prime}, s\right)=\left(\theta^{n} \omega^{\prime}, x-x_{n}(\omega)\right) \quad \text { if } \quad x_{n-1}(\omega) \leqq x<x_{n}(\omega),
$$

and for $x<0$

$$
T_{x} \omega=T_{x}\left(\omega^{\prime}, s\right)=\left(\theta^{n} \omega^{\prime}, x-x_{n}(\omega)\right) \quad \text { if } \quad x_{n}(\omega) \leqq x<x_{n+1}(\omega) .
$$


In the context of ergodic theory, such a flow is called the special flow under the ceiling function $1 / K$ and the basic automorphism $\theta$. Each $T_{x}$ preserves the measure $P$ (see [2]) and if $\theta$ is ergodic then $\left\{T_{x}\right\}$ is also ergodic. The last assertion follows from the observation that any $\left\{T_{x}\right\}$-invariant set $A \in \mathscr{F}$ is, up to a $P$-null set, of the following form: $A=\left\{\left(\omega^{\prime}, s\right): \omega^{\prime} \in A^{\prime}, 0 \leqq s<K\left(\omega^{\prime}\right)^{-1}\right\}$, where $A^{\prime} \in \mathscr{F}^{\prime}$ is a $\theta$-invariant set. Moreover, it is easy to see that $m_{T_{x} \omega}(\cdot)=m_{\omega}(\cdot+x)$ and $q_{T_{x} \omega}(\cdot)=q_{\omega}(\cdot+x)$. Thus we have constructed the framework of Sect. 2.

To finish the proof of Theorems $1^{\prime}$ and $2^{\prime}$, all we have to do is to check that the Ljapounov index $\gamma^{\prime}(\lambda)$ defined by (6.2) is a positive constant multiple of the Ljapounov index $\gamma(\lambda)$ defined in Sect. 2. Let $M_{\lambda}(x: \omega)$ be the matrix defined by (2.4). Then some elementary calculations show

$$
M_{\lambda}\left(x_{n}(\omega) ; \omega\right)=\left[\begin{array}{cc}
0 & 1 \\
K\left(\theta^{n+1} \omega^{\prime}\right) & -K\left(\theta^{n+1} \omega^{\prime}\right)
\end{array}\right] M_{\omega}^{\prime}\left(n ; \omega^{\prime}\right)\left[\begin{array}{cc}
1 & K\left(\omega^{\prime}\right)^{-1}-s \\
1 & s
\end{array}\right] .
$$

From this we have

$$
\begin{aligned}
\gamma(\lambda) & =\lim _{x \rightarrow \infty} \frac{1}{x} \log \left\|M_{\lambda}(x ; \omega)\right\|=\lim _{n \rightarrow \infty} \frac{1}{x_{n}(\omega)} \log \left\|M_{\lambda}\left(x_{n}(\omega) ; \omega\right)\right\| \\
& =\lim _{n \rightarrow \infty} \frac{n}{x_{n}(\omega)} \frac{1}{n} \log \left\|M_{\lambda}^{\prime}(n ; \omega)\right\|=\frac{1}{c} \gamma^{\prime}(\lambda) .
\end{aligned}
$$

Now let us turn to the proof of Theorem 3'. From the Lemma 5.2 and the construction of random measure $m_{\omega}$, we see that for $P$-a.e. $\omega \in \Omega$,

$$
h_{-}\left(\xi+i 0 ; T_{x_{n}(\omega)} \omega\right)=-\overline{h_{+}\left(\xi+i 0 ; T_{x_{n}(\omega)} \omega\right)} \text { a.e. } \xi \in A^{\prime}, n \in \mathbb{Z}^{1} .
$$

It is clear that if this relation holds for $\omega=\left(\omega^{\prime}, s\right)$ then it holds also for $\omega_{0}=\left(\omega^{\prime}, 0\right)$. Therefore an argument parallel to that of Theorem 3 shows that $P^{\prime}$-almost surely $\left\{\left(M\left(\theta^{j} \omega^{\prime}\right), K\left(\theta^{j} \omega^{\prime}\right)\right): j \geqq n\right\}$ is determined by $\left\{\left(M\left(\theta^{j} \omega^{\prime}\right), K\left(\theta^{j} \omega^{\prime}\right)\right): j<n\right\}$ for each $n$. This proves Theorem $3^{\prime}$.

\section{A Characterization of $\boldsymbol{L}_{\omega}=$ Laplacian}

It would be interesting to study the inverse correspondence between $\left\{L_{\omega}\right\}$ and $w$ functions. In this section we point out some simple facts related to this problem. The Corollary of Proposition 7.1 is an analogue of Kotani's Theorem 5.1. However, the proof is entirely different.

\section{Proposition 7.1}

(i) $\lim _{\lambda \downarrow-\infty}(-1 / \sqrt{-\lambda}) w(\lambda)=E\left[\int_{0}^{1} \sqrt{m_{\omega}^{\prime}(x)} d x\right]$, where $m_{\omega}^{\prime}(x)$ is the density of the absolutely continuous part of $m_{\omega}(d x)$.

(ii) If $q_{\omega}(x) \equiv 0$, then

$$
\lim _{\lambda \uparrow 0}(-1 / \sqrt{-\lambda}) w(\lambda)=\sqrt{E\left[m_{\omega}([0,1])\right]}
$$


Proof. (i) The result of S. Watanabe asserts, in our notations, that

$$
\lim _{\lambda \downarrow-\infty}(-1 / \sqrt{-\lambda}) \int_{0}^{1} h_{-}\left(\lambda ; T_{x} \omega\right) d x=\int_{0}^{1} \sqrt{m_{\omega}^{\prime}(x)} d x .
$$

(See [10], Appendix II, Proposition. Watanabe treats the case of $q \equiv 0$, but it is easy to extend his argument to the case where $q \neq \equiv 0$ and $q$ is bounded.) On the other hand, making use of the Riccati equation, we have

$$
\left|\int_{0}^{1} h_{-}\left(\lambda ; T_{x} \omega\right) d x\right|^{2} \leqq \int_{0}^{1} h_{-}\left(\lambda ; T_{x} \omega\right)^{2} d x=-\lambda m_{\omega}((0,1])+h_{-}\left(\lambda ; T_{1} \omega\right)-h_{-}(\lambda ; \omega) .
$$

Taking the expectation in (7.1) and changing the order of the limit and the integration, which is justified by (7.2) and (4.2), we obtain the desired conclusion.

(ii) First note that (2.1) and Y. Kasahara's result ([10] Corollary to Theorem 2.1 with $h(\lambda)$ there being replaced by $-h_{+}(\lambda)^{-1}$ ) imply

$$
\lim _{\lambda \uparrow 0}(-1 / \sqrt{-\lambda}) h_{+}(\lambda ; \omega)=\sqrt{E\left[m_{\omega}([0,1])\right]}
$$

with probability one. From Fatou's lemma, we have

$$
\sqrt{E\left[m_{\omega}([0,1])\right]} \leqq \frac{\lim }{\lambda \uparrow 0}(-1 / \sqrt{-\lambda}) w(\lambda) .
$$

On the other hand, by using the equality in (7.2), Schwarz's inequality and the stationarity of $P$,

$$
\begin{aligned}
{[(-1 / \sqrt{-\lambda}) w(\lambda)]^{2} } & =\frac{1}{-\lambda} E\left[\int_{0}^{1} h_{+}\left(\lambda ; T_{x} \omega\right) d x\right]^{2} \leqq \frac{1}{-\lambda} E\left[\int_{0}^{1} h_{+}\left(\lambda ; T_{x} \omega\right)^{2} d x\right] \\
& =E\left[m_{\omega}([0,1])\right] .
\end{aligned}
$$

The desired conclusion follows immediately from (7.3) and (7.4).

Corollary. Assume $q_{\omega} \equiv 0$. Then in order that there exists a non-random constant $a>0$ such that

$$
L_{\omega}=-a d^{2} / d x^{2} P \text {-a.e. } \omega,
$$

it is necessary and sufficient that $w(\lambda)=-c \sqrt{-\lambda}$ with some positive constant $c$.

Proof. The necessity of the condition is obvious. Suppose $w(\lambda)=-c \sqrt{-\lambda}$ with some $c>0$. Then Proposition 7.1 shows that

$$
E\left[\int_{0}^{1} \sqrt{m_{\omega}^{\prime}(x)} d x\right]^{2}=E\left[m_{\omega}([0,1])\right] .
$$

But by Schwarz's inequality, we have in general

$$
E\left[\int_{0}^{1} \sqrt{m_{\omega}^{\prime}(x)} d x\right]^{2} \leqq E\left[\int_{0}^{1} m_{\omega}^{\prime}(x) d x\right] \leqq E\left[m_{\omega}([0,1])\right]
$$

So (7.5) implies firstly that $m_{\omega}(d x)$ is absolutely continuous on $(0,1)$ with probability 
one and secondly that $m_{\omega}^{\prime}(x)$ is constant almost everywhere on $\Omega \times(0,1)$. By the stationarity this must hold almost everywhere on $\Omega \times \mathbb{R}^{1}$. Consequently $m_{\omega}(d x)$ is proportional to Lebesgue measure with a coefficient independent of $\omega$.

Remark. From (2.1) and the assumption $q_{\omega} \equiv 0$, it follows that inf $\Sigma=0$ (see [10] Appendix I). Hence by Lemma 5.1 of $[9], w(\lambda)=-c \sqrt{-\lambda}$ with some $c>0$ if and only if $\gamma(\xi)=0$, a.e. $\xi \in[0, \infty)$. For related topics, see Deift and Simon [4].

It is natural to look for the characterization for $-L_{\omega}$ to be equivalent to the Laplacian of difference type $-L_{0}:\left(-L_{0} u\right)_{n}=u_{n+1}+u_{n-1}-2 u_{n}$. Although the author could not solve this problem, the following is easily seen. Here we return to the general case where $q_{\omega}$ is not necessarily zero.

Proposition 7.2. Assume that $n(\xi)$ defined by (4.6) is bounded. Then with probability one, $\operatorname{Supp}\left(m_{\omega}\right)$ consists of isolated points only.

Proof. From the assumption and (4.5),

$$
\lim _{\lambda \downarrow-\infty}-\frac{d w}{d \lambda}(\lambda)=\lim _{\lambda \downarrow-\infty} \int_{0}^{\infty} \frac{-\lambda}{\xi-\lambda} d n(\xi)=\int_{0}^{\infty} d n(\xi)<\infty .
$$

On the other hand, it is not difficult to see that if $x \in \operatorname{Supp}\left(m_{\omega}\right)$, then

$$
-\lambda g_{\lambda}(x, x: \omega) \uparrow \int_{0-}^{\infty} \sigma_{11}\left(d \xi: T_{x} \omega\right)=\frac{1}{m_{\omega}[x]}, \quad \text { as } \lambda \downarrow-\infty,
$$

where both sides become infinite simultaneously if $m_{\omega}[x]=0$ (compare [5], Sect. 5.7(3)). Applying the monotone convergence theorem to Lemma 4.3, we get

$$
\int_{0}^{\infty} d n(\xi)=E\left[\int_{(0,1]} \frac{1}{m_{\omega}[x]} m_{\omega}(d x)\right] .
$$

This implies firstly that $m_{\omega}(d x)$ consists of point masses only, and secondly that $E\left[\#\left\{x \in(0,1]: m_{\omega}[x]>0\right\}\right]<\infty$.

Acknowledgement. The author warmly thanks Professor S. Kotani and Professor T. Shiga who led him to the theme of this work and gave him useful suggestions which greatly simplified the proofs. He also thanks Professor I. Kubo and Professor H. Totoki who pointed out his mistake in the proof. The rewriting of this paper was completed during the author's stay at the ETH Zürich. He warmly thanks Professor H. Föllmer, Professor J. Moser and the other staff members, in the ETH for their hospitality, valuable discussions and encouragement. He also expresses his thanks to Professor K. Uchiyama who, reading through his manuscript, helped him rewrite this paper, and to the referee who gave the author valuable comments.

\section{References}

1. Carmona, R.: Random Schrödinger operators. École d'Été de Probabilités de Saint-Flour 1984 (to appear)

2. Cornfeld, I. P., Fomin, S. V., Sinai, Ya. G.: Ergodic theory. Berlin, Heidelberg, New York: Springer 1982

3. Craig, W., Simon, B.: Subharmonicity of Ljapounov index. Duke Math. J. 50, No. 2, 551-560 (1983)

4. Deift, P., Simon, B.: Almost periodic Schrödinger operators III. The absolutely continuous spectrum in one dimension. Commun. Math. Phys. 90, 389-411 (1983)

5. Dym, H., McKean, H. P.: Gaussian processes, function theory and the inverse spectral problems. 
New York, San Francisco, London: Academic Press 1976

6. Johnson, R., Moser, J.: The rotation number for almost periodic potentials. Commun. Math. Phys. 84, 403-438 (1982)

7. Kac, I. S., Krein, M. G.: R-functions-analytic functions maping the upper halfplane into itself. Am. Math. Soc. Trans. (2) 103, 1-18

8. Kingman, J. F. C.: Subadditive processes. École d'Été de Probabilités de Saint-Flour 1975. Lecture Notes in Mathematics, vol. 539, pp. 167-223 Berlin, Heidelberg, New York: Springer 1976

9. Kotani, S.: Ljapounov indices determine absolutely continuous spectra of random one-dimensional Schrödinger operators. Proc. Taniguchi Symp. SA Katata (1982), 225-247

10. Kotani, S., Watanabe, S.: Krein's spectral theory of strings and generalized diffusion processes. Lecture Notes in Mathematics, vol. 923, pp. 235-259 Berlin, Heidelberg, New York: Springer 1982

11. McKean, H. P.: Elementary solutions for certain parabolic partial differential equations. Tran. Am. Math. Soc. 82, 519-548 (1956)

12. Pastur, L. A.: Spectral properties of disordered systems in the one-body approximation. Commun. Math. Phys. 75, 179-196 (1980)

13. Simon, B.: Kotani theory for one dimensional stochastic Jacobi matrices. Commun. Math. Phys. 89, 227-234 (1983)

Communicated by B. Simon

Received September 14, 1984; in revised form September 12, 1985 